\title{
Modelling and Experimentation on Mechanical Properties of Graphene-Oxide Cement
}

\author{
Zhiyuan Lin $^{1}$, Ding Fan ${ }^{1}$, and Shangtong Yang ${ }^{1}$ \\ ${ }^{1}$ Department of Civil and Environmental Engineering, University of Strathclyde, Glasgow, G1 1XJ, United Kingdom
}

\begin{abstract}
Cementitious nano-composites have recently attracted considerable research interest in order to improve their properties such as strength and durability. Graphene oxide (GO) is being considered as an ideal candidate for enhancing the mechanical properties of the cement due to its good dispersion property and high surface area. Much of work has been done on experimentally investigating the mechanical properties of GO-cementitious composites; but there are currently no models for accurate estimation of their mechanical properties, making proper analysis and design of GO-cement based materials a major challenge. This paper attempts to develop a novel multi-scale analytical model for predicting the elastic modulus of GO-cement taking into account the GO/cement ratio, porosity and mechanical properties of different phases. This model employs Eshelby tensor and Mori-Tanaka solution in the process of upscaling the elastic properties of GO-cement through different length scales. In-situ micro bending tests were conducted to elucidate the behavior of the GO-cement composites and verify the proposed model. The obtained results showed that the addition of GO can change the morphology and enhance the mechanical properties of the cement. The developed model can be used as a tool to determine the elastic properties of GO-cement through different length scales.
\end{abstract}

\section{Introduction}

Nanomaterials have recently attracted considerable attention for their application to cementitious composites, for improving the properties of cement/concrete. A wide range of nanomaterials such as metal oxide or silica, nanofibers, nanotubes and graphene have been added into cementitious materials to improve their compressive and flexural strength, Young's modulus and other microstructure properties. Graphene is a single atom thick sheet of hexagonally arranged carbon atoms with a carbon-carbon distance of $0.142 \mathrm{~nm}$ [1]. It is about more than 100 times stronger than steel by weight, conducting heat and electricity efficiently and nearly transparent [1]. However, there is an issue of dispersion when mixing with cementitious materials. Graphene oxide (GO), a product of chemical exfoliation of graphene, can be an excellent nano reinforcement for cementitious nanocomposites, due to its good dispensability in water, high aspect geometric ratio and excellent mechanical properties.

Previous studies have revealed that GO shows better adhesion bonding properties to the matrix than graphene, as a result of its oxygen functional groups provided [2, 3]. Moreover, GO is hydrophilic due to the carbonyl, epoxide, carboxyl and hydroxyl groups on its surface thus making it highly dispersible in water as a result [4]. Because of these functional groups, GO can react with cement and provide strong stress transfer capacity which enables higher stress transfer between the matrix and the
GO, thereby increasing the stiffness of the plain matrix [5]. Literature review suggests that GO can increase the 28-day compressive and flexural strength of cement by as much as $72.7 \%$ and $67.1 \%$, respectively at a GO concentration of $0.06 \mathrm{wt} \%$ and $0.04 \mathrm{wt} \%$, respectively [5]. Saafi et al. [6] investigated the material and mechanical properties of grapheme/fly ash geopolymeric composite cement and experimentally quantified the enhancement in these properties.

This paper attempts to experimentally investigate the properties of GO-cement and develop a multi-scale analytical model for the elastic modulus of the GO reinforced cement, which is a key material parameter representing the elasticity of material. Volume fractions and mechanical properties of different phases at various length scales are considered in the formulation of the macroscale Young's modulus. Voids have also been considered in the formulation by assuming zero stiffness of them. In-situ SEM three-point bending tests were carried out to understand the fracture mechanisms of GO-cement composites and verify the derived model by comparing the Young's modulus obtained from both modeling and experimental tests. Mercury Intrusion Porosimetry (MIP) tests are also carried out to find out the effects of adding GO on the porosity of cement. Some key parameters such as the bulk and shear moduli of GO and C-S-H were chosen and their effects on the macroscale Young's modulus have been investigated. The developed model can be used as a useful tool for predicting the Young's modulus of GO-cement with a 
variety of composition and fractions. Accurate prediction of the mechanical properties of GO-cement will help accelerate the application of GO into civil engineering industries.

\section{Experiment Setup}

In-situ SEM three-point bending test was performed by Tungsten Filament Scanning Electron Microscope incorporated with three-point bending testing facility. Load-deflection curves were determined from the test which was used to determine the Young's modulus of the GO cement samples, as well as morphology of GO cement at different scales. GO was obtained from market and the original concentration of GO was $4 \mathrm{mg} / \mathrm{ml}$. The chemical composition of GO was Carbon 49-56\%, Hydrogen $0-1 \%$, Nitrogen $0-1 \%$, Sulfur $0-2 \%$ and Oxygen $41-50 \%$. The GO cement composite was prepared by mixing cement, water and GO. The dosage of GO was $0.00 \%, 0.02 \%$ and $0.035 \%$ by weight. Water/cement ratio used was 0.4. For dispersion of GO in water, bath sonicator was used for 30 minutes for each $\mathrm{GO} /$ water solution with different dosages of GO. The cement was then added and the mixture was stirred. The fresh GO cement composite was poured into a $6 \mathrm{~mm} \times$ $10 \mathrm{~mm} \times 40 \mathrm{~mm}$ moulds for casting and curing. In the first 24 hours the specimens were kept in environmental chamber with constant temperature of $20^{\circ} \mathrm{C}$; after that, the specimen were removed from the moulds and cured in water at $20^{\circ} \mathrm{C}$ for 28 days. Some GO cement samples were coated with gold for better conductivity in light of the SEM test. The testing arrangement and the geometric parameters are illustrated in Figure 1. Displacement is applied in the middle of the beam and loading speed is $0.05 \mathrm{~mm} / \mathrm{min}$.
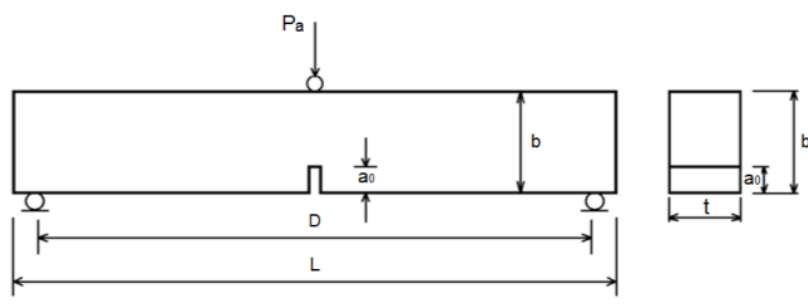

Fig. 1. The Schematic of three-point bending test.

In the three-point bending test, the whole loading process up to failure of the specimens can be divided into three parts: linear elastic, nonlinear plastic and fracture failure. To calculate the Young's modulus of each specimen, only the elastic deformation phase was chosen, which is associated to the initial loading period. The Young's modulus E can be determined as follows:

$$
E=\frac{P_{a} D^{3}}{4 t\left(b-a_{0}\right)^{3} d}
$$

where $P_{a}$ is the load, $D$ is the distance between the two supports, $t$ is the width of the specimen, $b$ is the depth of specimen, $a_{0}$ is the notch and $d$ is the deflection of specimen. The values for these parameters are $L=40 \mathrm{~mm}, b=10 \mathrm{~mm}, t=6 \mathrm{~mm}, a_{0}=1 \mathrm{~mm}$ and $D=36 \mathrm{~mm}$.

\section{Modelling}

In this paper, GO-cement composites are studied at 3 different length scales, as shown in Figure 2. At microscale, there are mainly $\mathrm{GO}$ and $\mathrm{C}-\mathrm{S}-\mathrm{H}$ while at mesoscale, calcium hydroxide $(\mathrm{CH})$, voids and some unhydrated cement $[7,8]$. To determine the scales in multi-scale modelling, the structural dimensions need to be significantly larger than the dimensions of the material in homogeneities. Accordingly, Representative Volume Element (RVE) is introduced to represent the basic unit of homogeneity. Eshelby tensor and MoriTanaka solution are employed in the process of upscaling the elastic properties of GO-cement through different length scales.

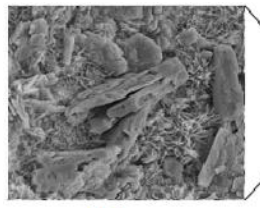

(a)

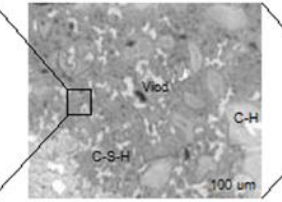

(b)

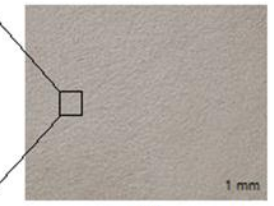

(c)
Fig. 2. The scale range of GO cement (a) Microscale (b) Mesoscale (c) Macroscale.

There is an argument that the GO is a disk-like material while Mori-Tanaka may not be directly applicable. However, in practice, a few layers of GO always stack together or fold, forming a roughly spherical shape, as shown in Figure 3. It is almost impossible to have a single layer of disk-like GO sheet in cement and the application of Mori-Tanaka scheme can therefore be justified.
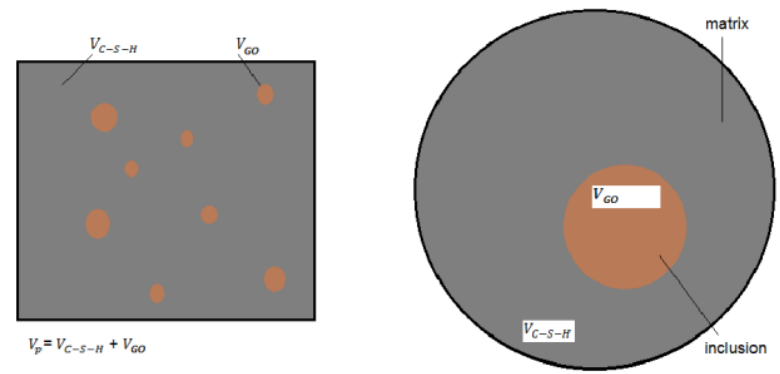

Fig. 3. The proposed RVE and matrix-inclusion geometry for GO cement.

RVE is utilized which is essential to find the homogenized constitutive response at each smaller scales. In this paper, an RVE with a size of $50 \times 50 \mu \mathrm{m}$ is chosen for the two-phase matrix-inclusion geometries at the microscale level. At this level, the REV is displayed in Figure 3. The effective Young's modulus after homogenization $E_{h}$ can be derived as follows, 


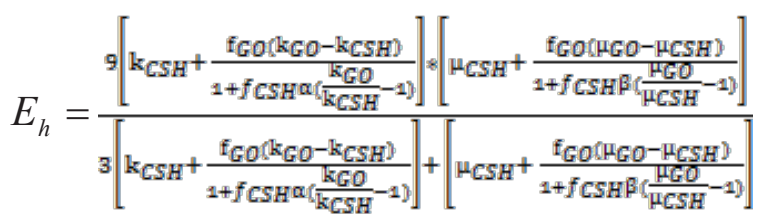

\section{Results}

The microstructures of the specimens for different GO dosages are presented in Figure 4. Figure 4(a) shows the crack tip of the notch for zero GO inclusion in cement. Figure 4(b) illustrates the morphology for $0.02 \% \mathrm{GO}$ inclusion in which, the GO sheets hold and bind cement together. In Figure 4(c), it shows a piece of grouped GO on the surface with ettringite binding the edge. However, in Figure 4(b) and Figure 4(c), there is no similar flower or crystal formation as found in some other studies. The reason why cement shows the rock-like surface on GO is probably because of the absence of Polycarboxylic superplasticizer. Polycarboxylic superplasticizer ensures the GO spreads across uniformly in the water so that GO can be mixed with cement randomly and forms different crystalline shapes. However, there is lack of evidence as if Polycarboxylic superplasticizer has effect on formation and properties of GO cement composite.

The cracked surfaces for the samples with different percentages of GO have also been shown in Figure 5. It is interesting to find that the fracture surface for zero GO cement is relatively smoother with little ettringite presenting in the crack. The fracture surface for $0.035 \%$ GO cement shows rough surface around the GO, whose length is ranging from $3 \mu \mathrm{m}$ to $30 \mu \mathrm{m}$. The rough surface might probably be caused by the existence of GO. The GO can be clearly seen in C-S-H tends to react with the functional groups of $\mathrm{GO}$ and the chemically-bound interface produced offers stronger binding mechanism in terms of the covalent bond between oxygen in GO and calcium in cement [21]. Such a chemically-bound cementitious nanocomoposite at the interfaces can enhance the mechanical performance of cement by transferring higher stress at the interfaces.

The averaged load-deflection curves from the threepoint bending test for GO inclusion of $0.00 \%, 0.02 \%$ and $0.035 \%$ respectively are shown in Figure 6 . Clearly the GO inclusion of $0.035 \%$ samples show highest peak load and largest slope which represents the stiffness of the samples. The averaged response for each batch appears to have roughly the same displacement at which the peak load reached. All the samples have very brittle failure, i.e., sudden drop of the load after its peak value. This is because the test is not designed to capture the post-peak fracture behavior of the GO cement which requires closed-loop testing system with crack mouth opening displacement being the control signal for loading.


Fig. 4. SEM pictures of (a) $0.00 \%$ GO cement (b) $0.02 \%$ GO cement and (c) $0.035 \%$ GO cement.

To demonstrate the application of the derived analytical model, an example is undertaken by using the same inputs from the experiments. The values of basic variables of the materials are presented in Table 1 . With the values of these basic variables, the homogenized Young's modulus of GO cement can be calculated based on the model derived. To verify the developed analytical model, the results are compared with those from the experiments. It can be seen in Table 2, the result from the model and the test are in good agreement. For $0.02 \%$ 
of GO inclusion by weight, the Young's modulus of cement determined from the test increases from 11.63 $\mathrm{GPa}$ to $12.63 \mathrm{GPa}$ and from the model increases to 12.99GPa. The enhancement in Young' modulus is $8.6 \%$ and $11.7 \%$ respectively. The difference could be partially due to the assumption that the interfaces between GO and cement are perfectly bound.



(a)

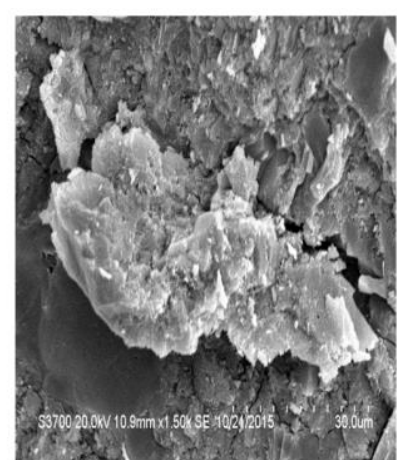

(b)
Fig. 5. The fracture surface for (a) $0.00 \%$ GO cement (b) $0.035 \%$ GO cement.

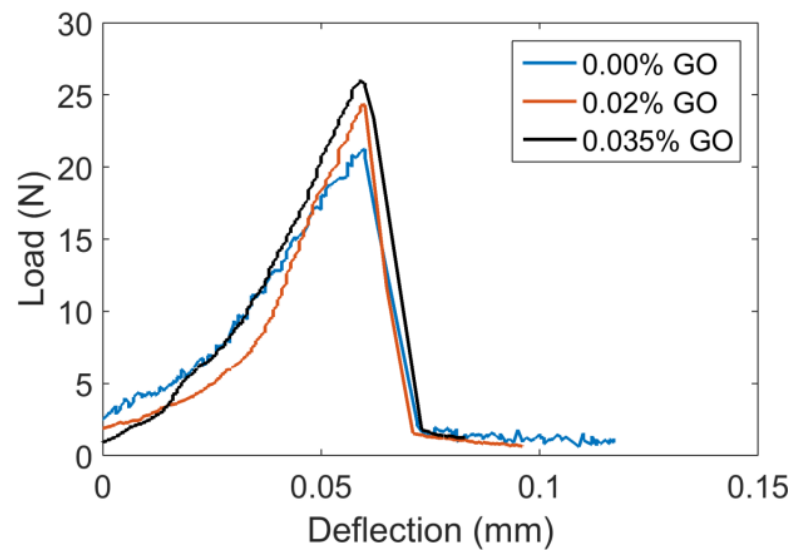

Fig. 6. Load-deflection curves obtained from the three-point bending test for different percentages of GO additions.

Table 1. Material properties for GO and C-S-H as input for the model

\begin{tabular}{|c|c|c|c|}
\hline Properties & GO & C-S-H & CH \\
\hline Young's Modulus E & $350 \mathrm{GPa}$ & $11 \mathrm{GPa}$ & $38 \mathrm{GPa}$ \\
\hline Possion's ratio v & 0.197 & 0.24 & 0.31 \\
\hline
\end{tabular}

Table 2. Comparison of the results from the experiments and the model

\begin{tabular}{|c|c|c|c|c|}
\hline \multicolumn{2}{|c|}{ Weight Fraction of GO } & $0 \%$ & $0.02 \%$ & $0.035 \%$ \\
\hline \multirow{4}{*}{ Experiment } & Young's & 11.63 & 12.63 & 14.64 \\
& Modulus & $\mathrm{GPa}$ & $\mathrm{GPa}$ & $\mathrm{GPa}$ \\
\cline { 2 - 5 } & Improvement & $/$ & $8.6 \%$ & $25.9 \%$ \\
\hline \multirow{3}{*}{ Model } & Young's & 11.63 & 12.99 & $\begin{array}{c}14.89 \\
\mathrm{GPa}\end{array}$ \\
\cline { 2 - 5 } & Modulus & $\mathrm{GPa}$ & $\mathrm{GPa}$ & \\
\cline { 2 - 5 } & Improvement & $/$ & $11.70 \%$ & $28.0 \%$ \\
\hline
\end{tabular}

\section{Conclusions}

In this paper in-situ three-point bending SEM test was undertaken to determine the Young's modulus of GO cement experimentally and the results have been compared with the analytical model. It has been found that the results from the derived model and the experimental tests are in good agreement. The analytical model developed can predict the elastic mechanical performance of GO cement by considering all possible scales. It has been found that the existence of GO can significantly change the morphology and substantially enhance the mechanical properties of the cement. The developed model can be used as a useful tool to evaluate the global elastic mechanism of GO cement based on the microscale mechanical behavior of the materials.

\section{References}

1. Hancock, Y. The 2010 nobel prize in physicsground-breaking experiments on graphene. Journal of Physics D: Applied Physics 2011, 44, 473001.

2. Yang, X.; Tu, Y.; Li, L.; Shang, S.; Tao, X.-m. Welldispersed chitosan/graphene oxide nanocomposites. ACS applied materials \& interfaces 2010, 2, 17071713.

3. Hashin, Z. Analysis of composite materials-a survey. Journal of Applied Mechanics 1983, 50, 481505.

4. Qiu, L.; Yang, X.; Gou, X.; Yang, W.; Ma, Z.F.; Wallace, G.G.; Li, D. Dispersing carbon nanotubes with graphene oxide in water and synergistic effects between graphene derivatives. Chemistry-A European Journal 2010, 16, 10653-10658.

5. Lv, S.; Ting, S.; Liu, J.; Zhou, Q. Use of graphene oxide nanosheets to regulate the microstructure of hardened cement paste to increase its strength and toughness. CrystEngComm 2014, 16, 8508-8516.

6. Saafi, M.; Tang, L.; Fung, J.; Rahman, M.; Liggat, J. Enhanced properties of graphene/fly ash geopolymeric composite cement. Cement and Concrete Research 2015, 67, 292-299.

7. Hashin, Z. The elastic moduli of heterogeneous materials. Journal of Applied Mechanics 1962, 29, 143-150.

8. Kouznetsova, V.; Brekelmans, W.; Baaijens, F. An approach to micro-macro modeling of heterogeneous materials. Computational Mechanics 2001, 27, 37-48. 